\title{
Experimental Results of 802.11a Wireless LAN System over Optical Fiber
}

\author{
I. Haroun, F. Gouin, L. Boucher, and L. Bouchard \\ Communications Research Centre Canada (CRC), \\ Ottawa, Ontario, Canada K2H 8S2 \\ \{ibrahim.haroun, francois.gouin, luc.boucher, luc.bouchard\} @erc.ca
}

\begin{abstract}
In this paper, the rationale of using wireless over optical fiber to support broadband wireless deployments is presented and initial measured results derived from a proof-of-concept experiment are described. The primary objective of this project is to investigate the technical feasibility of using an optical/wireless infrastructure capable of delivering broadband multi-media traffic to and from subscribers. In such a scheme, the optical fibers are used to route the broadband optically modulated signals to distribution centers, where the RF signals get detected and wirelessly transmitted to each remote user. An experimental test setup using an 802.11a access point over optical fiber was developed in order to evaluate the potential of this technology merge.
\end{abstract}

\section{Introduction}

The initial objective of this project was to explore strategies to provide high capacity mobile broadband wireless access for the future, enabling the support of a wide range of applications under different propagation and mobility environments. Some of the limitations involved in providing broadband wireless access in a mobile environment are described in the background section of this paper. The rationale of using wireless over optical fiber as part of the solution to implement mobile broadband communications is then presented. In section 3, a possible architecture for wireless over optical fiber transmission is described. In order to evaluate the potential of the solution presented (the RF and optical fiber technologies merge), an experimental proof-of-concept setup using an 802.11a access point over optical fiber was put in place and measurements were conducted. This is reported in section 4. Conclusions and future work are presented in section 5.

\section{Background}

\subsection{Limitations in Providing Broadband Wireless Access}

One of the key issues in broadband wireless access is the ability to transmit high peak data rates to individual terminals. Apart from the obvious requirement of having enough spectrum space, there are a few fundamental limitations on how high the data 
rate can go over wireless links. Factors influencing the data rate include RF power, signal distortion, mobility, etc. [1-2]:

- RF Power: Reliable data transmission requires a certain minimum amount of energy for each binary bit of information. Then, transmitting data faster requires higher power. Some of the methods that can be employed to increase the average received power include: transmitting more power, reducing the distance between the transmitter and receiver and/or using higher antenna gain.

- Signal Distortion: In a multipath radio propagation environment, the receiver intercepts several copies of each transmitted symbol. As the symbol transmission rate increases, the symbol duration gets shorter and consequently the different copies of a symbol overlap with other symbol causing the wellknown phenomenon of Inter-Symbol-Interference (ISI). The ISI problem cannot usually be corrected by increasing the power. It can be corrected to some extent by employing equalization techniques or techniques such as spread spectrum (e.g. CDMA -Code Division Multiple Access) or frequency division multiplexing (e.g. OFDM - Orthogonal Frequency Division Multiplexing), each technique having its limitations.

- Mobility: Another physical problem is the ability of the system to support terminal mobility. If terminals are allowed to move from location to location, then the system must be able to provide coverage over the service area.

The key requirements to providing broadband wireless access in urban areas include the needs to support: very high density of terminals, high data rate per unit area, various modes (e.g. fixed, nomadic and mobile) and the requirement to be economically deployable on a large scale $[2,3]$.

\subsection{Why RF over Optical Fiber?}

A broadband wireless solution in urban areas with wide range of radio services must support fixed, nomadic and mobile terminals. In order to maintain high data rates everywhere and be spectrum efficient, the radio cells must be small [3]. The small cells translate into a very large number of base stations. Hence, for a successful deployment, it is necessary to have economical and effective transport and distribution schemes. With such a large number of base stations, the fiber distribution loop becomes an attractive solution, with the following supporting justifications [2,3]:

- One of the main justifications is to combat the physical limitations of large cells. Having small cells allows one to: (1) shorten the distance between bases and mobiles; (2) reduce the signal distortion by reducing the range of delay spread and scattering and (3) provide an effective means of deploying site-specific radio coverage and also (4) facilitate effective growth strategies.

- With a large number of cells (or base station antennas), the wireless over optical fiber concept solves two problems: (1) it reduces the cost of base stations since many functions can be removed from the base station and placed at some central processing locations. The huge bandwidth capacity of the fiber is used to communicate these functions between the processing units and the base stations 
and (2) the use of fiber greatly simplifies the communications among base stations.

- Since the wireless over optical fiber concept allows for removing most of the intelligence from the base stations and placing them at a few central points, better management of the radio resources is possible. Algorithms can be developed to match the radio resources to cell requirements and to adaptively change the radio resource allocations among adjacent cells.

- One of the key advantages of the "wireless over optical fiber" concept is the fact that fiber optic local distribution is gaining considerable momentum. Cable and other wired access operators are cooperating to extend the fiber optic broadband capacity as close as possible to the end user. The concept is termed: "fiber-tothe-curb". If and when this happens, the wireless operators could use advantageously this metropolitan fiber infrastructure to support large-scale deployments for mobile broadband access.

The use of wireless over fiber is not a new concept. Since the first investigations of optical-RF interactions several decades ago, the applications now cover areas such as antenna remoting, optically-controlled phased array antenna systems, and microwave signal processing. However, the optical and optoelectronic components used for optically distributing RF signals have in the past been very expensive and, as a result, the technology has only been used in very limited deployments. Advances in fiber optic technology now provide more affordable modulating laser devices and optical detectors.

These developments lead to the possibility of utilizing wireless over optical fiber to carry wireless LAN (WLAN) signals, therefore enabling the delivery of broadband multi-media traffic to and from subscribers on a large scale [3,4]. An experimental test setup using 802.11a access points over optical fiber was developed in order to demonstrate the potential of this technology merge. The test set-up and the measured results are described in Section 4.

\section{Wireless over Optical Fiber Architecture}

Figure 1 illustrates the infrastructure of a single cell of a WLAN over optical fiber system. In this scheme, the base station (also called access point) transceiver of each cell is equipped with an Electric-to-Optic converter $(\mathrm{E} / \mathrm{O})$ in the receive path, and an Optic-to-Electric converter $(\mathrm{O} / \mathrm{E})$ in the transmit path. A possible architecture for multiple base stations using wireless over optical fiber transmissions is illustrated in Figure 2. All the digital signal processing functions such as modulation and demodulation, and frequency assignment are performed at the central office.

In the downstream path (from the access point to the customer), an optical detector is required to convert the optical signal to an RF signal. The RF signal will need to be amplified in order to support the needed EIRP (Effective Isotropic Radiated Power). For example, the 802.11a standard [5,6] for North America uses three power levels for its $300 \mathrm{MHz}$ bandwidth, the first $100 \mathrm{MHz}(5.15-5.25 \mathrm{GHz})$ is restricted to a maximum output power of $50 \mathrm{~mW}$, the second $100 \mathrm{MHz}(5.25-5.35 \mathrm{GHz})$ is restricted to maximum power of $250 \mathrm{~mW}$, and the third $100 \mathrm{MHz}(5.725-5.825 \mathrm{GHz})$ is restricted to a maximum output power of 1 Watt for outdoor applications. The 
802.11a standard for North America also specifies channel spacing of $20 \mathrm{MHz}$ and guard band spacing of $30 \mathrm{MHz}$ at the band edges in order to meet FCC (US) and IC (Canada) spectral mask requirements.

One of the main advantages of 802.11a systems is its robustness against multi-path fading and increased spectrum efficiency because of the use of OFDM modulation [7,8]. WLAN 802.11a systems currently provide high-speed Internet access (up to 54 Mbps) for indoor environments such as public buildings, shopping malls, airports, and similar applications. These systems can be modified to support larger coverage outdoor applications.

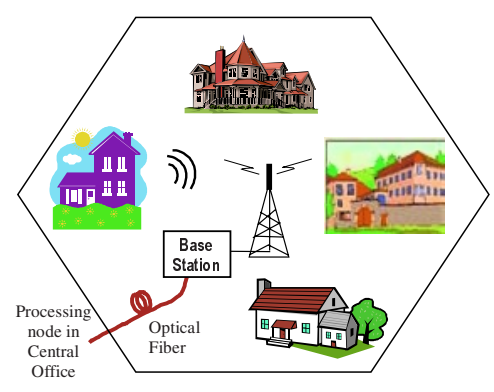

Fig. 1. Single Cell Architecture

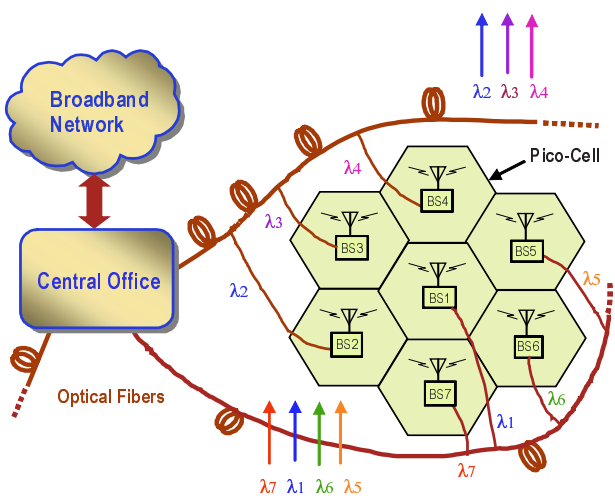

Fig. 2. Possible Architecture for Multiple Cells

\section{Proof-of-Concept}

\subsection{Optoelectronic Components Selection}

The 802.11a WLAN standard operates in the frequency range between 5.150 and $5.825 \mathrm{GHz}$. A few sources and photo-receivers that were relatively easily available and capable of operating over that frequency range were evaluated. These fiber optic links were first evaluated in terms of transmission as a function of frequency. A network analyzer was used for these measurements, as shown in Figure 3. The optical link transmission response of one of the source/photo-receiver selected for this experimentation (the HP834 series) is shown in figure 4. The transmission of the optical link was measured over the range from $400 \mathrm{MHz}$ to $10 \mathrm{GHz}$. As seen, the RF transmission is attenuated by approximately $8.5 \mathrm{~dB}$ at $5 \mathrm{GHz}$ and decreases smoothly to $-10 \mathrm{~dB}$ at $6 \mathrm{GHz}$, the range of interest for 802.11a. Of course, the variation in the $\mathrm{RF}$ transmission is even smaller over any given $802.11 \mathrm{a}$ RF channel, which is only 20 $\mathrm{MHz}$ wide. The losses are mainly due to the conversion from RF to optical and back to RF. 


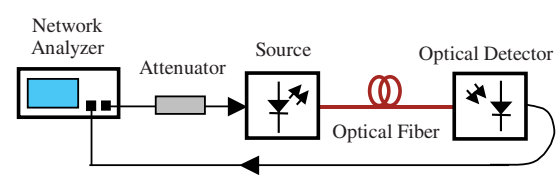

Fig. 3. Setup to Measure Transmission Response

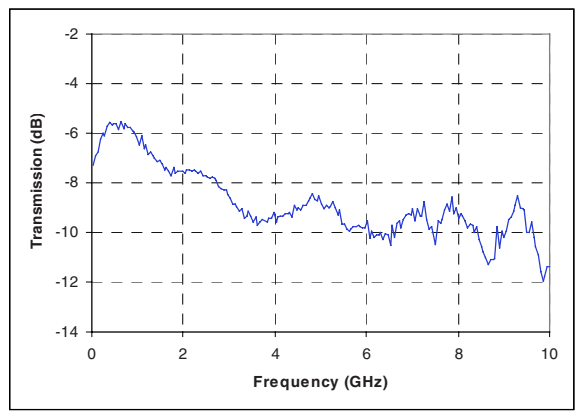

Fig. 4. Optical Link Transmission Response

\subsection{Experimental Test Set-up and Measured Results}

The goal of the initial experiments was to determine whether data transmission using the 802.11a WLAN standard could tolerate the distortions caused by a fiber optic link, particularly those caused by the laser. In this setup, an 802.11a access point (AP) was connected to an optical link that consisted of a laser, a two-meter span of single mode fiber, and an optical detector, as seen in figure 5 below. Since the actual losses on the single mode fiber are small, typically $<1 \mathrm{~dB} / \mathrm{km}$, the losses of this type of optical link are primarily due to conversion at its end points. As a result, the two-meter span should give a good indication of some of the degradations that can be expected from the optical conversion.

The laser used was an "HP 83403C Lightwave source" emitting at $1.5 \mu \mathrm{m}$ and the detector was an "HP 83411D Lightwave receiver". Both are specified as having a bandwidth of $300 \mathrm{MHz}$ to $6 \mathrm{GHz}$. The 802.11a access point was a D-Link DWL5000AP.

In order to establish a baseline for this experiment, the RF spectrum of the signal transmitted from the access point towards the optical link was first measured. The access point (AP) was configured for 54 Mbps transmission rate and $5.28 \mathrm{GHz}$ channel frequency. As shown in figure 5, the output of the optical detector was connected to a spectrum analyzer to monitor the 802.11a signal at the output of the access point (1) and after transmission over the fiber (2). Figure 6 compares these 2 points, i.e. the measured spectrum at the input and output of the 2-meter optical link.

From the measured results, one observes a drop in the signal level by about $8.5 \mathrm{~dB}$ in the central part of the $20 \mathrm{MHz}$ channel. This drop is in agreement with the response of the HP 834 series optical link shown previously in Figure 4. One can also observe that there is no significant degradation in the shape of the channel response.

Figure 7 shows the RF spectrum at $1 / 2$ meter distance away from the transmitting antenna (test set-up shown in figure 5 (3)). The measured results indicate a drop in the signal level due to the propagation path-loss, but the shape of the channel spectrum did not degrade. With increasing distance between the transmitting and receiving antennas, the shape of the RF spectrum starts to degrade. In order to compensate for the optical link losses and to maintain the system required BER, an RF amplifier will be required to boost the signal level at the output of the optical link. 


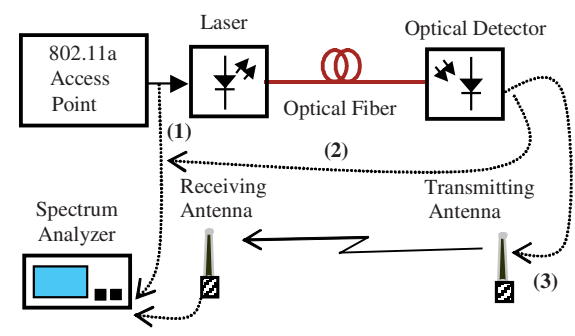

Fig. 5. 802.11a over Optical Fiber Test Setup

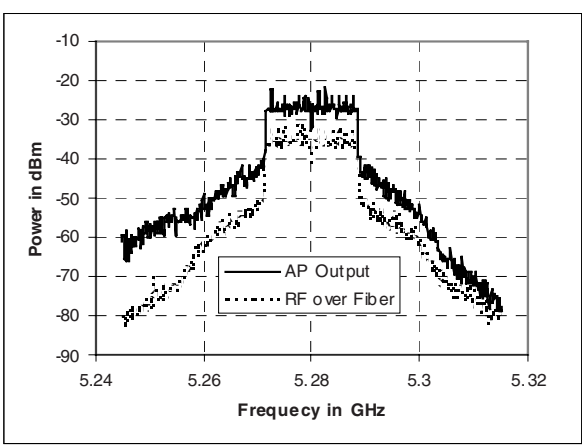

Fig. 6. RF Spectrum before and after Transmission over the Optical Link

Finally, the spectrum analyzer shown in the test set-up of Figure 5 (3) was replaced with a laptop computer equipped with an 802.11a card, in order to establish a link with another laptop computer at a remote distance. Data files transfer between the access point and the remote laptop computer were established and successful.

\subsection{Bi-directional Optical Fiber Link}

Once the successful transmission of 802.11a over optical fiber was demonstrated, another optical-link was added in the reverse direction. A Miteq MDD optical link was used for the up-stream link because the source includes a pre-amplifier which amplifies the received RF signal before modulating the laser thereby improving the signal-to-noise ratio. The down-stream link used the HP 834 series as described before. Using this test set up, successful transfer of data files to and from the access point, via optical links was demonstrated. This experiment demonstrated that the optical links were able to successfully transmit and receive WLAN 802.11a signals.

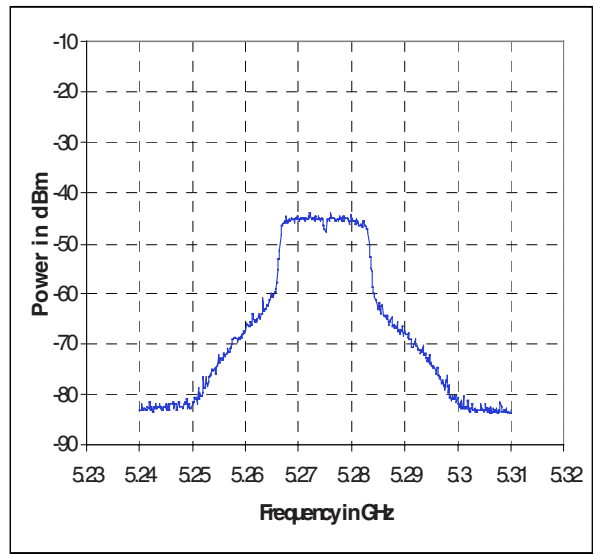

Fig. 7. RF Spectrum at $1 / 2$ meter Distance from the Transmitting Antenna

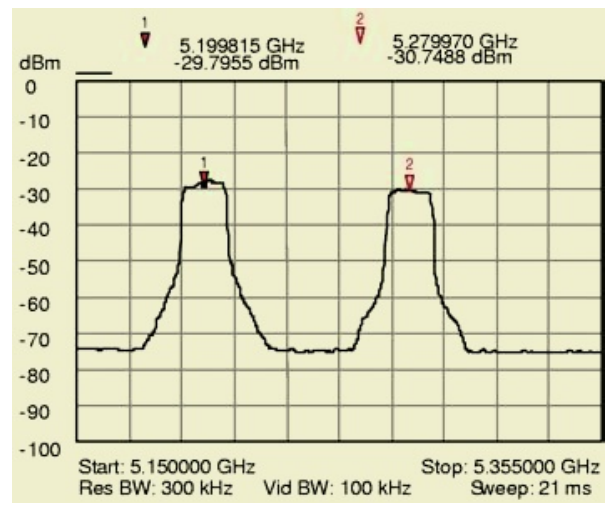

Fig. 8. Two-OFDM Channels - RF Spectrum at the Output of the Optical Link 
The bit-error-rate (BER) is typically used to characterize the quality of a digital link. It is difficult to measure the BER directly when using an access point (AP) because the error handling is buried in the transfer protocol. One approach would be to do away with the AP and cardbus adapter combination (to generate the RF signal) and replace them by a vector signal generator capable of producing an OFDM signal. A vector spectrum analyzer could then be used to display the signal after the transmission. This experiment would further characterize this system, and is planned for the future.

\subsection{Multichannel WLAN over a Single Fiber-Optic Link}

In order to determine that the link is free of intermodulation products, the impact of transmitting more than one RF channel over a single optical wavelength was investigated. In this experiment, one channel was generated by an 802.11a access point (D-Link DWL-5000AP) and the other channel was obtained from an Agilent E4438C Vector Signal Generator (using the Agilent 802.11a signal studio software). The two channels were combined and applied to an optical link (Miteq MDD). The output of the optical link was fed to a spectrum analyzer to investigate any intermodulation that might occur due to carrying multiple channels. The measured spectrum at the output of the optical link is shown in Figure 8. As seen the resulting spectrum showed no apparent inter-modulation distortion when two OFDM signals were carried over the optical link.

\section{Conclusions and Future Work}

Initial measured results using 802.11a WLAN over optical fiber demonstrates that the 802.11a signals can be transmitted over optical fiber without causing undue degradation to the signal characteristics. Merging of the optical fiber and the WLAN (802.11a) technologies could provide a good solution for both the indoor and outdoor broadband access, while improving spectrum efficiency and robustness against multipath fading. Also, it could provide a significant cost reduction and simplification to the wireless base-station deployments for future generation wireless systems such as $4 \mathrm{G}$. Additional work is needed and is planned to complement these results, in order to demonstrate the full feasibility of this approach.

\section{References}

[1] P. Larijani and R.H.M. Hafez, "Power assignment and access control for multimedia CDMA wireless networks" IEEE transactions on Veh. technology, 1999.

[2] Hafez, R., "Universal Broadband Wireless Access", Internal Report, Communications Research Centre (CRC), Ottawa, Canada, December 2001.

[3] I. Haroun, G. Chan, R. Hafez, L. Bouchard, L. Boucher, "Feasibility Study of Radio Over Optical Transmission Systems for Increased System Efficiency", IEEE Int. Symp. on Electromagnetic Compatibility EMC 2003, 11-16 May 2003, Istanbul, Turkey. 
[4] Al-Raweshidy, H., Komaki, S., "Radio over Fiber Technologies for Mobile Communications Networks", Norwood, MA, Artech House, 2002.

[5] http://standards.iee.org/getieee802/

[6] Bob O'Hara, Al Petrick “The IEEE 802.11 Handbook: a Designer's Companion”, IEEE Press, 1999.

[7] Heiskala, J., Terry, J., "OFDM Wireless LANs: A Theoretical and Practical Guide ", SAMS Publishing, 2002.

[8] Van Nee, R., Prasad, J., "OFDM For Wireless Multimedia Communications", Artech House Publishing, 2000. 